\section{Myocardial Positron Emission Tomography/Computed Tomography Scan Revealing Right Coronary Artery Involvement in Large Vessel Vasculitis}

To the Editor:

We describe a 55-year-old woman who presented with epigastric pain radiating to the back, chest discomfort, and fever. Blood tests showed increased level of acute-phase reactants. Computed tomography angiography (CTA) revealed a partially thrombized aneurysm of the superior mesenteric artery and stenosis of the celiac trunk and inferior mesenteric artery. Arteriography confirmed the stenoses. Two angioplasties were successfully performed, leading to prompt resolution of the abdominal pain. She was then treated with acetylsalicylic acid $100 \mathrm{mg} /$ day and clopidogrel 75 $\mathrm{mg} /$ day. Nevertheless, her chest pain worsened, she developed a troponinnegative crescendo angina, and was diagnosed with a non-ST-elevation acute coronary syndrome. Cardiac angiography showed normal coronary arteries. Myocardial blood flow was assessed with a combined positron emission tomography-computed tomography (PET/CT) and ${ }^{13} \mathrm{~N}$-ammonia. A stress-rest protocol with dipyridamole was used (Figure 1).

Regional ischemia was detected in the inferoapical and inferior wall, indicating mild hemodynamically significant involvement of the right coronary artery. Left ventricular ejection fraction was normal (73\%). Assessment of hypermetabolic foci was performed with a whole-body $\mathrm{PET} / \mathrm{CT}$ scan and ${ }^{18} \mathrm{~F}$-fluorodeoxyglucose (FDG; Figure 2). Increased tracer uptake (maximum standardized uptake value 3.1) was revealed in the ascending aorta, left subclavian, left common carotid, pulmonary and innominate arteries, and less intense uptake in the thoracic, descending, and abdominal aorta. On the basis of clinical signs, laboratory findings, CTA, and PET/CT scans, the patient was diagnosed with Takayasu arteritis (TA). She was given prednisone $50 \mathrm{mg} /$ day with a tapering schedule and methotrexate $15 \mathrm{mg} / \mathrm{week}$, achieving complete resolution of symptoms. One year later a FDG-PET/CT scan did not reveal any vascular uptake.

TA is a primary large vessel vasculitis of unknown origin that mainly affects the aorta and its branches and is more common in young women. Coronary involvement in TA is not rare, being reported in $7 \%$ to $29 \%$ of cases, and is associated with high disability and mortality ${ }^{1}$. Such involvement presents in the form of stenosis, complete obstruction, aneurysm, or coronary steal syndrome. Stenosis or occlusion of the coronary ostium and the proximal segments of the coronary arteries is most frequently detected. Narrowing of the coronary arteries seems to be caused by progression of inflammatory process through the intima, media, and adventitia, leading to fibrosis of the vessel wall ${ }^{2}$.

Invasive coronary angiography is the clinical gold standard for the diagnosis of coronary artery stenosis and allows immediate treatment with angioplasty. However, it can have complications and may fail to visualize early changes of the coronary arteries.

The use of noninvasive techniques to assess coronary involvement in patients with TA is growing ${ }^{3}$. FDG-PET has been used in the diagnosis of large vessel vasculitis and, although in part still debated, has a promising role in monitoring disease activity and response to treatment $t^{4,5,6}$. Coronary CTA has been developed into a clinically useful method for visualization of coronary artery stenosis with high negative predictive value, but the

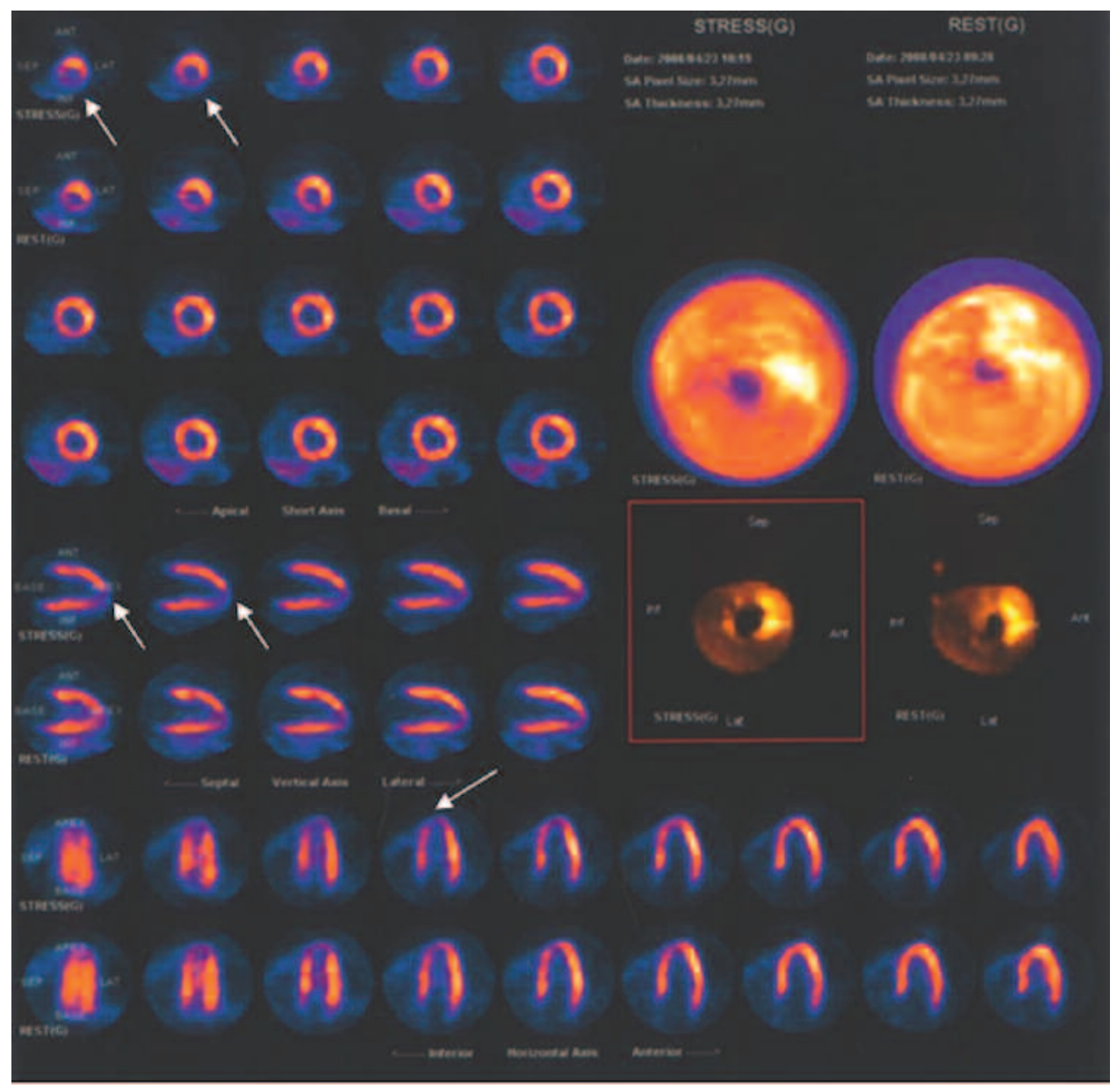

Figure 1. Myocardial perfusion PET/CT images with ${ }^{13} \mathrm{~N}$-ammonia at stress and rest showing ischemia (arrows) in the right coronary artery regions involving the inferoapical and inferior wall. 
evaluation of stenosis is often uncertain ${ }^{7}$. The PET/CT imaging technique allows a combination of angiography and perfusion imaging in short, quantitative, low-dose radiation protocols and provides information about anatomy and function of arteries ${ }^{8}$. Perfusion imaging alone could not always separate microvascular disease from epicardial stenoses but PET/CT significantly improved this accuracy, allowing noninvasive detection of coronary artery disease in symptomatic patients as well as the detection and characterization of atherosclerotic plaque ${ }^{9,10}$.

To our knowledge, this is the first description of coronary artery involvement in TA detected using a PET/CT perfusion scan in a symptomatic patient with normal coronary angiography. This case indicates that cardiac PET/CT perfusion imaging could be a useful noninvasive tool for the early diagnosis and quantitative determination of cardiac perfusion abnormalities in the assessment of active coronary involvement secondary to TA, even earlier than morphologic changes appear on conventional imaging such as CTA and angiography.

MATTEO PIGA, MD, Chair of Rheumatology and Rheumatology Unit, University and AOU of Cagliari, Cagliari; LIVIA RUFFINI, MD, Nuclear Medicine Unit, AOB "San Michele", Cagliari, current affiliation Imaging Department, AOU Parma, Parma; CARLO SALVARANI, MD,
Rheumatology Unit, Arcispedale Santa Maria Nuova, Reggio Emilia; ALESSANDRO MATHIEU, MD, Chair of Rheumatology and Rheumatology Unit, University and AOU of Cagliari, Cagliari, Italy. Address correspondence to Dr. M. Piga, Rheumatology Unit, University Clinic AOU, SS 554 - 09042 Monserrato, Cagliari, Italy. E-mail: matteopiga@alice.it

\section{REFERENCES}

1. Rav-Acha M, Plot L, Peled N, Amital H. Coronary involvement in Takayasu's arteritis. Autoimmun Rev 2007;6:566-71.

2. Matsubara O, Kuwata T, Nemoto T, Kasuga T, Numano F. Coronary artery lesions in Takayasu arteritis: Pathological considerations. Heart Vessels Suppl 1992;7:26-31.

3. Qureshi M, Bacilio D, Bhatia K, Robi A, Einstein AJ, Bokhari S The role of noninvasive imaging in the diagnosis and management of Takayasu's arteritis with coronary involvement. J Nucl Cardiol 2009;16:995-8.

4. Arnaud L, Haroche J, Malek Z, Archambaud F, Gambotti L, Grimon $\mathrm{G}$, et al. Is (18)F-fluorodeoxyglucose positron emission tomography scanning a reliable way to assess disease activity in Takayasu arteritis? Arthritis Rheum 2009;60:1193-2000.

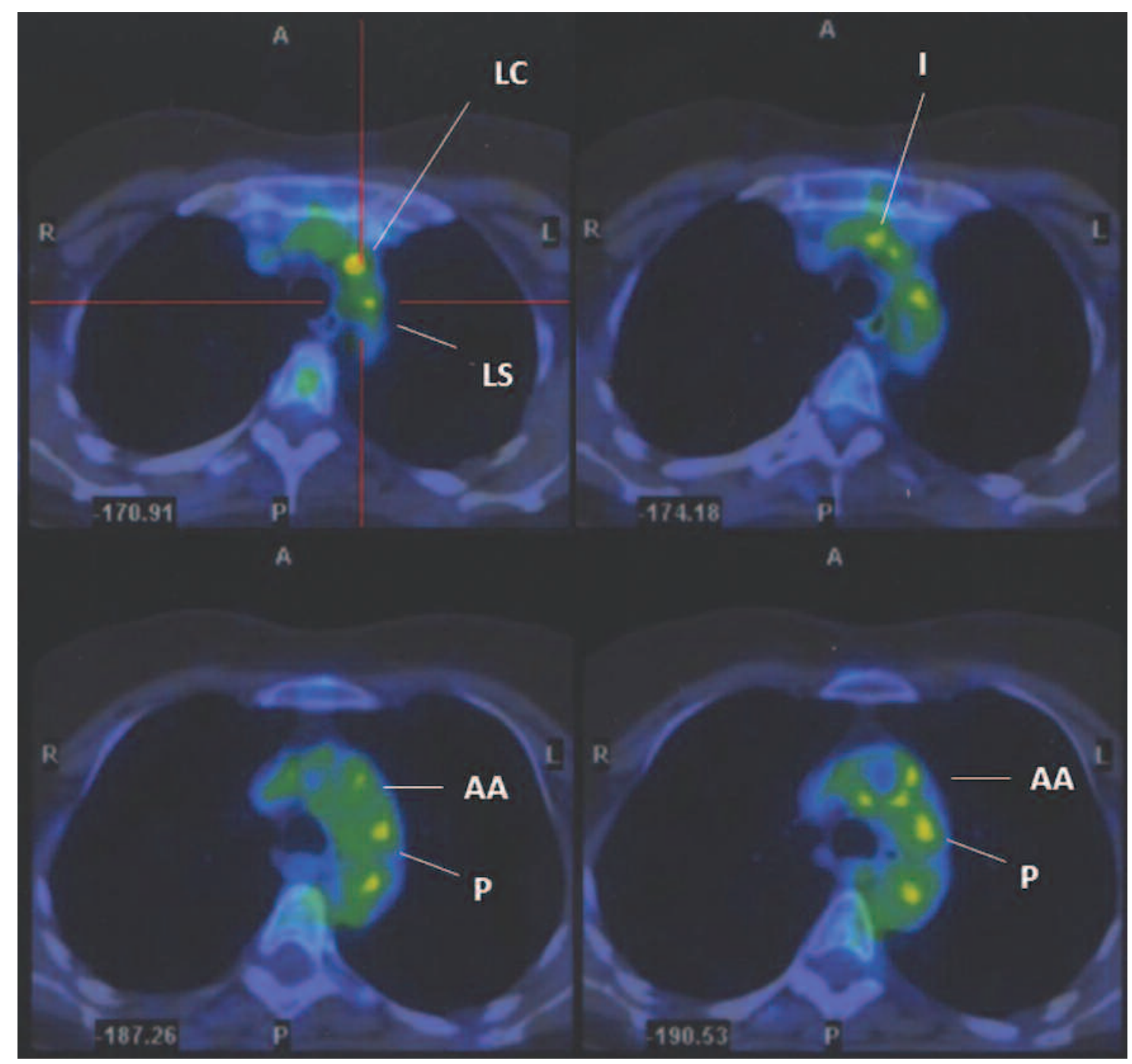

Figure 2. PET/CT images with ${ }^{18} \mathrm{~F}-\mathrm{FDG}$ showing hypermetabolic foci in the ascending aorta (AA), left subclavian (LS), left common carotid (LC), pulmonary (P), and innominate (I) arteries. 
5. Lee KH, Cho A, Choi YJ, Lee SW, Ha YJ, Jung SJ, et al. The role of (18)F-fluorodeoxyglucose-positron emission tomography in the assessment of disease activity in patients with Takayasu arteritis. Arthritis Rheum 2012;64:866-75.

6. Lehmann P, Buchtala S, Achajew N, Haerle P, Ehrenstein B, Lighvani $\mathrm{H}$, et al. 18F-FDG PET as a diagnostic procedure in large vessel vasculitis - A controlled, blinded re-examination of routine PET scans. Clin Rheumatol 2011;30:37-42.

7. Soto ME, Meléndez-Ramírez G, Kimura-Hayama E, Meave-Gonzalez A, Achenbach S, Herrera MC, et al. Coronary CT angiography in Takayasu arteritis. JACC Cardiovasc Imaging 2011;4:958-66.

8. Balink H, Houtman PM, Collins J. 18F-FDG PET versus PET/CT as a diagnostic procedure for clinical suspicion of large vessel vasculitis. Clin Rheumatol 2011;30:1139-41.
9. Kajander S, Joutsiniemi E, Saraste M, Pietilä M, Ukkonen H, Saraste A, et al. Cardiac positron emission tomography/computed tomography imaging accurately detects anatomically and functionally significant coronary artery disease. Circulation 2010;122:603-13

10. Stolzmann P, Subramanian S, Abdelbaky A, Maurovich-Horvat P, Scheffel H, Tawakol A, et al. Complementary value of cardiac FDG PET and CT for the characterization of atherosclerotic disease. Radiographics 2011;31:1255-69.

J Rheumatol 2012;8; doi:10.3899/jrheum.120353 